\title{
P-062I - Testosterone therapy for Sexual Dysfunction in Men with Type 2 Diabetes: A Systematic Review and Meta-analysis of Randomised Controlled Trials
}

\author{
Authors: M.Algeffari*(I,2), C. N. Jayasena* (I,3), P. MacKeith (4),A.Thapar (5),W. S. Dhillo (I), N. Oliver (I)
}

I Department of Investigative Medicine, Hammersmith Hospital, Imperial College London, London, UK.

2. Department of Family Medicine, College of Medicine, Qassim University, Saudi Arabia

3. Department of Andrology, Hammersmith Hospital, London, UK

4. Department of Public Health, University of East Anglia, Norwich, UK

5. Department of Surgery \& Cancer, Imperial College London, London, UK

Background: A growing number of randomised controlled trials (RCTs) have investigated the effectiveness of TT to improve sexual function in men with type 2 diabetes (I-8). However, none of these trials are large enough to dictate clinical consensus guiding whether affected patients should receive TT. Furthermore, many clinicians are cautious regarding the potential risks of cardiovascular disease and prostate cancer during TT (9).

Aim: To evaluate the effectiveness of testosterone therapy (TT) on a range of sexual function domains in men with type 2 diabetes.

Method: Electronic databases were searched for studies investigating the effect of TT on sexual function in men with type 2 diabetes. All randomised control trials (RCTs) were considered for inclusion if they compared the efficacy of TT with placebo and reported sexual function outcomes. Statistical analysis was performed using a random effects model and heterogeneity expressed as the 12 statistic.

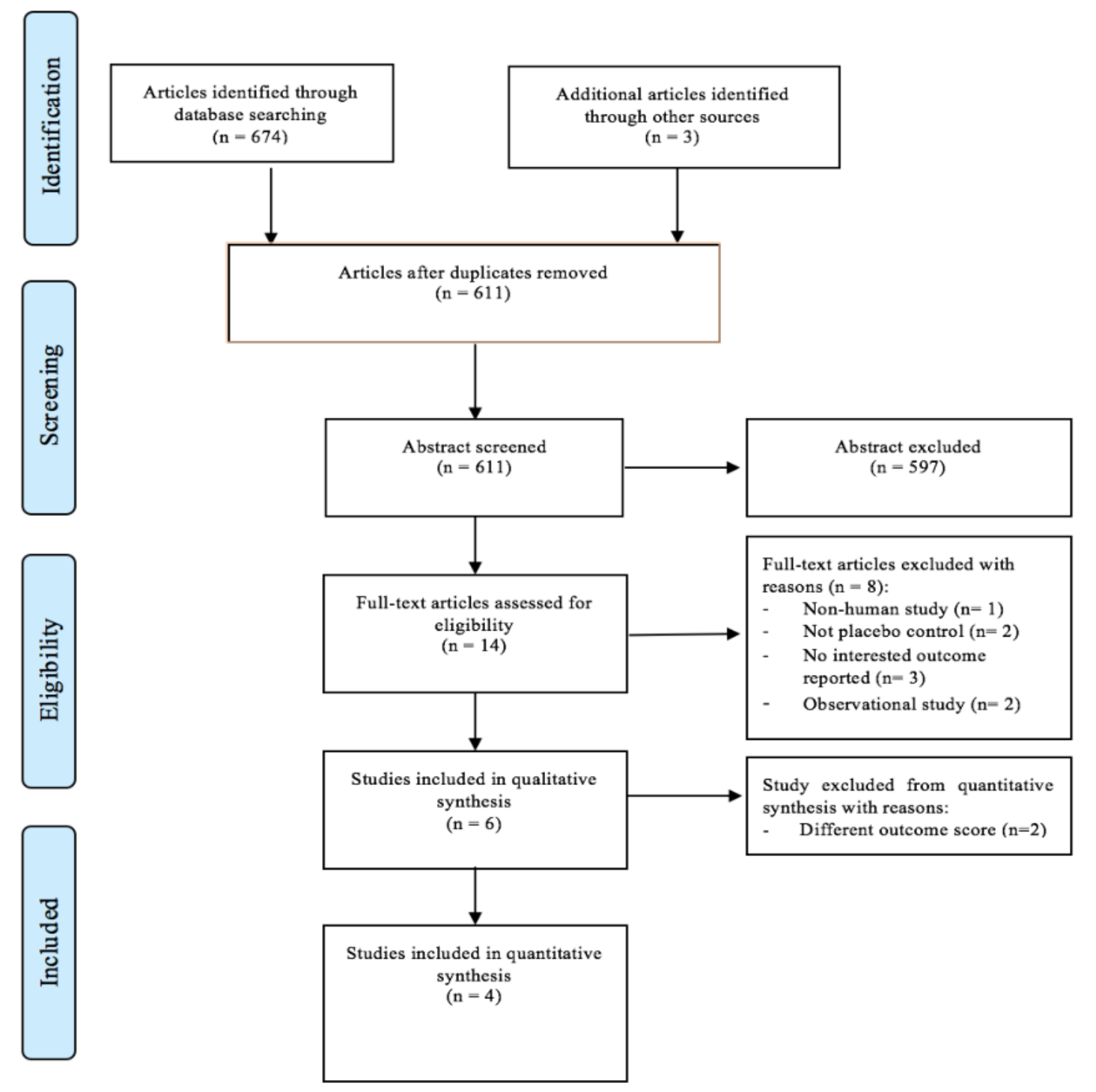

Results: A total of 6II articles were screened. Six RCTs inclusive of 587 men with type 2 diabetes were eligible for inclusion. The pooled data suggested that TT improves sexual desire (random-effects pooled effect size, 0.314; 95\% confidence interval [ $\mathrm{Cl}], 0.082-0.546)$ and erectile function (random-effects pooled effect size, 0.203; 95\% confidence interval [Cl], 0.007-0.399) when compared with control group.

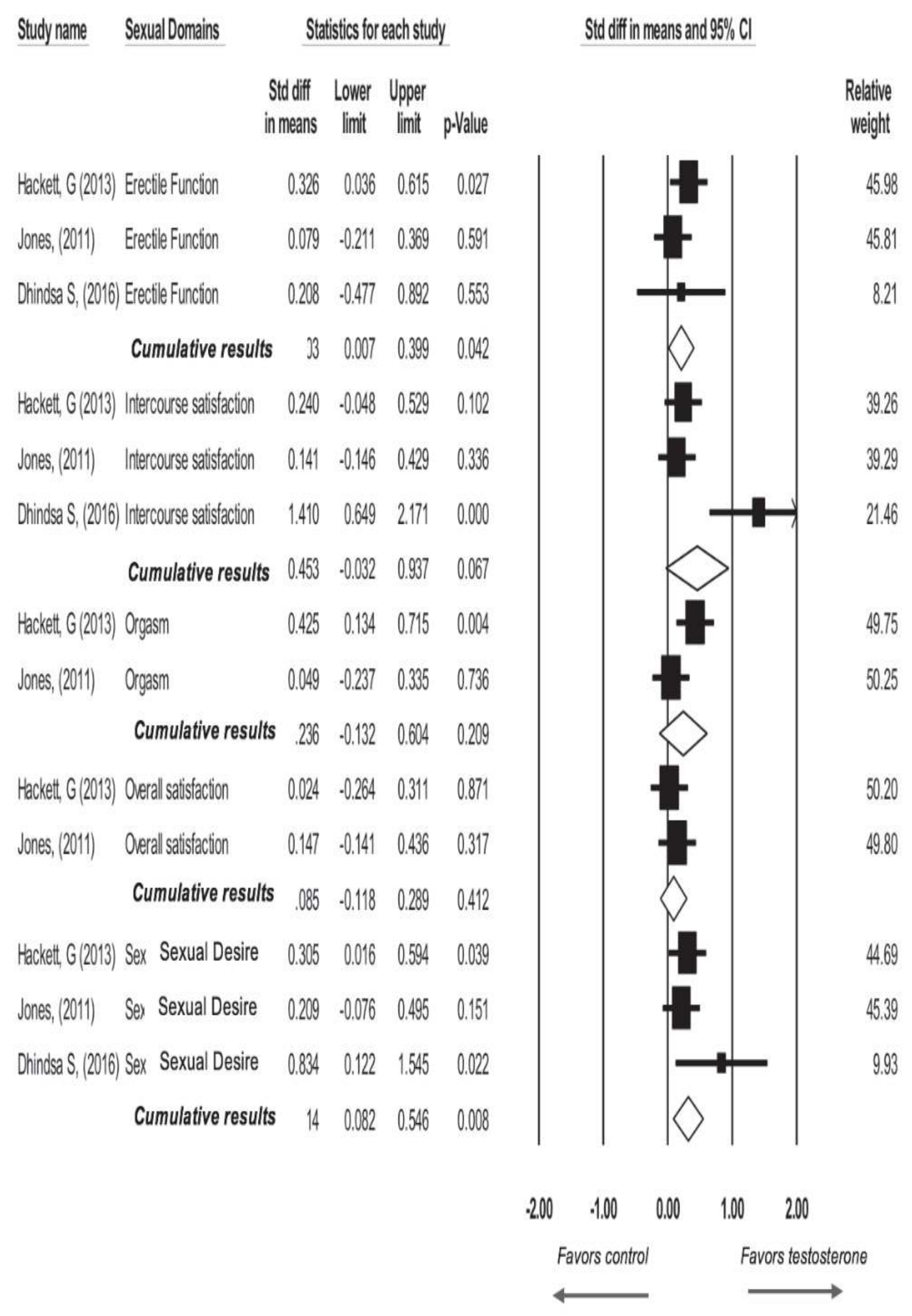

TT had no significant effect on constitutional symptoms or other sexual domains compared with control groups. No previous studies have investigated the incidence of prostate cancer, fertility and cardiovascular disease following TT in men with type 2 diabetes.

Conclusion: TT may moderately improve sexual desire and erectile function in men with type 2 diabetes; however, available data are limited, and the long-term risks of TT are not known in this specific patient group. We conclude that TT is a potential treatment for men with type 2 diabetes non-responsive to phosphodiesterase- 5 inhibitors. TT could be considered for men with type 2 diabetes when potential risks and benefits of therapy are carefully considered, and other therapeutic options are unsuitable. 